\title{
Artificial Lighting System in Outdoor Image Analysis Application for Oil Palm Fresh Fruit Bunches
}

\author{
N.M.A.Harun ${ }^{1}$, Z.Abdul Halim², and H.Ibrahim ${ }^{3}$
}

\begin{abstract}
In the application of outdoor image analysis for oil palm fresh fruit bunches (FFB) ripeness classification, color properties of the captured FFB images is constantly altered due to irregular outdoor lighting environment. In this research, an artificial lighting system is developed to control light intensity around the oil palm FFB before the image is captured for ripeness classification. The system consists of a light source which is LED and a light sensor which is LDR. Light sensor will measure the ambient lighting and automatically regulates the intensity of the artificial lighting according to the ambient lighting. The total of 540 images for four categories of ripeness, which are unripe, under-ripe, ripe and overripe are captured and analyzed. Three types of lighting modes, which are Off, Auto and On are used during the images acquisition. Hue values are extracted from every image. At an effective distance, which is 11 inches between the oil palm FFB and the artificial light source, the distinction of maturity level for unripe, under-ripe, ripe and overripe can be improved. By using the Auto lighting mode, the system can save power consumption by $22.5 \%$.
\end{abstract}

Keywords - Fruit ripeness classification, outdoor image analysis, artificial lighting and oil palm fresh fruit bunches.

\section{INTRODUCTION}

$I^{1}$ $\mathrm{N}$ the conventional method of FFB harvesting, the FFB maturity levels is determined by observing the FFB color and counting the amount of loose fruitlets on the ground [1]. While this method is widely practiced in local oil palm plantations, it was reported about $30 \%$ of local smallholders still harvest unripe oil palm FFB due to the lack of experience in evaluating the FFB maturity levels [2]. To reduce the reliance on the experienced harvester, the application of machine vision system for oil palm automation need to be initiated.

Several studies on the application of machine vision system for oil palm automation were already investigated and promising results in classifying the oil palm FFB maturity levels had been reported [3][4][5]. However, most of the studies were developed to perform under control lighting environment, which is contrary to the outdoor lighting

N.M.A.Harun ${ }^{1}$ is with the Universiti Sains Malaysia as a researcher.

Z.Abdul Halim ${ }^{2}$ is with Universiti Sains Malaysia. She is now in Electrical\&Electronic Engineering Department under Electronic Programme.

H.Ibrahim $^{3}$ is with the Electrical\&Electronic Engineering Department, Universiti Sains Malaysia. environment with variations in lighting intensity. Previous studies of oil palm automation system had reported a range of alteration in color properties of the captured oil palm FFB images due variations in outdoor lighting intensity and irregular shades from the oil palm canopy. Jamil, [11] had reported that the accuracy of ripeness of oil palm FFB had reduced to $27 \%$ in their experiment of FFB color grading conducted in the outdoor lighting environment. In a study of outdoor FFB image analysis developed by Ismail and Razali [6], an alteration in the color component of the captured FFB images had been reported due to variations in the outdoor lighting intensity. All of these report are concurring with the report by [3] in their study, that the hue values of the captured oil palm images alter as much as $13 \%$ to $42 \%$ for a small change in illumination intensities during the oil palm fruit inspection.

Besides, the phenomena of sunny, the shaded region that is generated by cloud transitions also contributes to the occurrence of color alteration in outdoor image analysis application. In early research [7], five color charts namely magenta, white, yellow, cyan, and red had shifted to blue once the color charts were moved from sunny to shaded place. In their following study [8] they had described on this effect as a hue shift phenomena, that is a cloudy skylight will shift the incident light on the surface of the object from a white light to a bluish light.

In the application of indoor image analysis for oil palm ripeness evaluation, the use of artificial lighting is extensively applied in order to provide a uniform lighting for oil palm color analysis [9][3][4][10][5]. Since the utilization of artificial lighting in the previous studies had demonstrated a promising results in classifying the oil palm FFB maturity levels, further research need to be carried out in order to develop a proper artificial lighting system to assist in classifying the oil palm FFB maturity levels in outdoor lighting environment.

Several researchers had reported a range of alteration in color properties of the captured oil palm FFB images due to variations in outdoor lighting intensity and irregular shades of the oil palm canopy [11][16]. Study about the use of artificial lighting to improve outdoor image analysis application has been investigated since late 1980's [12]. By incorporating the artificial lighting to their robotic fruit harvester, they had reported that between $45 \%$ and $93 \%$ of 
the target image were correctly classified without the assistance of any optical filter. In the following study conducted by Blandini and Levi [13], they had managed to detect about $70 \%$ of the visually perceptible fruit by utilizing an artificial lighting of $3200 \mathrm{~K}$ color temperature in detecting a hue reference of a citrus fruit. Singh [14] had applied an artificial lighting in the form of camera flash to their reconfigurable robotic vehicle known as Autonomous Prime Movers, APM and managed to improve the ability of the detection algorithm in green apple classification. The use of camera flash also had effectively reduced the size of glaring area in a study of outdoor sweet cherries color rating conducted by Wang [15], with an overall classification accuracy of over $85 \%$. Encouraging results delivered by various researchers [12][13][16][14][15] promotes the possibility of incorporating the artificial lighting to assist with outdoor oil palm image analysis application.

While the use of artificial lighting has shown to improve the outdoor image analysis applications, the continuous use of artificial lighting will significantly consume power. This study aimed to develop an optimized artificial lighting system for outdoor oil palm image analysis application. The system consists of LDR-based daylight responsive LED dimming system. The study was conducted in the oil palm plantation to simulate the actual outdoor lighting variations encountered by harvester. A few experiments have been conducted in this study. Three different artificial lighting modes are investigated which are On mode, Auto mode and Off mode. The On and the Auto modes are used to identify the amount of energy savings by implementing the LDR-based daylight responsive LED dimming system whereas the Off mode is used to investigate on the effect of outdoor lighting variations to oil palm FFB image analysis application.

\section{EXPERIMENTAL SETUP}

The hardware architecture of the proposed artificial lighting system consists of 5 main components, which are light source (LED), sensors (LDR), microcontroller, camera, and laptop computer. The block diagram of the complete hardware architecture for the proposed outdoor lighting system is shown in Fig. 1.

The LED, LDR, and network camera are placed at the tree top for inspection of the oil palm FFB. The LDR will measure the ambient lighting in the oil palm FFB surrounding. The measured analog signal of the LDR in electrical resistance is then converted to electrical voltage by using voltage divider circuit.

The Bridgelux BXRA C0361 white LED with a color temperature of $5600 \mathrm{~K}$ is chosen as the artificial light source for the proposed lighting system of this research. At the color temperature of $5600 \mathrm{~K}$, the color spectrum of the LED is comparable to the measured color temperature of a noon sunlight, which is at $5500 \mathrm{~K}$ (Stoppee \& Stoppee, 2009). The use of Bridgelux BXRA C0361 white LED as the source of artificial lighting delivers a comparable lighting performance to the 20-40 Watt of the incandescent light source, and 20-35 Watt of the halogen light source at only 4-8 Watt of energy consumption [17].

In order to test the proposed artificial lighting system, a field test is conducted in the actual oil palm plantation located in Universiti Sains Malaysia, Engineering Campus, Nibong Tebal Pulau Pinang. The height of palm oil tree in this area is around 20 feet, hence 20 feet of pole is used to capture the images of FFB. The images are captured by using the AXIS M1034W network camera in the size of $480 \times 610$ pixels in 24-bit of RGB color space. Four different categories of images are captured which are unripe, underripe, ripe and overripe.

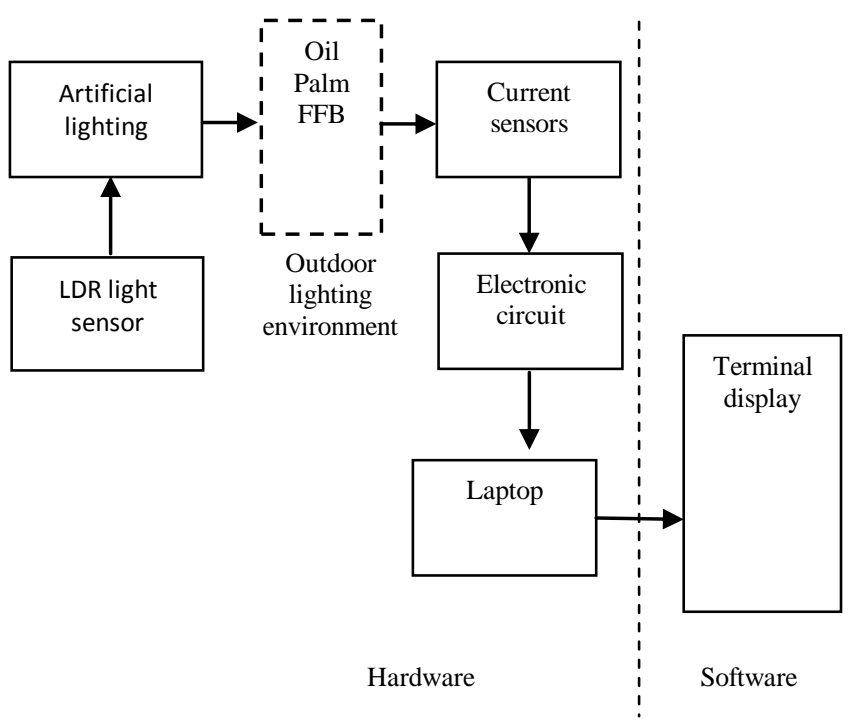

Fig. 1 Hardware architecture of the proposed outdoor lighting system

Three artificial lighting modes are proposed in the experiments, which are Auto, On and Off. Images are captured every one hour from 8.00am until 4.00pm. Five samples from each category from different trees are collected. Since there are three modes of lighting system, four categories of ripeness, nine intervals time and five tress, hence the total of 540 images are captured in this experiment.

To investigate on the effect of artificial lighting to oil palm FFB images, all captured images have to go through the segmentation process in order to extract their corresponding fruit color properties for FFB ripeness determination. All FFB images are captured by using the AXIS M1034W network camera in the size of $480 \times 610$ pixels in 24-bit of RGB color space. There are two color regions on the oil palm FFB which are fruit colors, and redundant colors contributed by unnecessary elements such as spikes, empty sockets, palm fibers, and palm fronds. In this research, the captured FFB image is segmented into two different regions of fruit colors and redundant colors by using the existing image segmentation software developed by Fadilah [18][19][20] in the previous research.

Hue color properties is selected for image analysis in this research since it provides reliable discrimination capabilities in discriminating the oil palm FFB colors. The color pixels of 
the segmented oil palm FFB image is converted to hue values of HSI (hue, saturation, and intensity) color model.

\section{RESULTS}

A graph of hue histogram from both segmented images are shown in Fig. 2. From the observation, the effect of blue ambient lighting generated from the skylight is perceivable at bin 55 to 71 of the hue histogram. It is observed that the FFB images captured at 8:00 am contains higher amount of blue colored hue properties with 53 pixels at bin 61 , while image captured at 12:00 pm contains only 17 pixels at bin 61 .

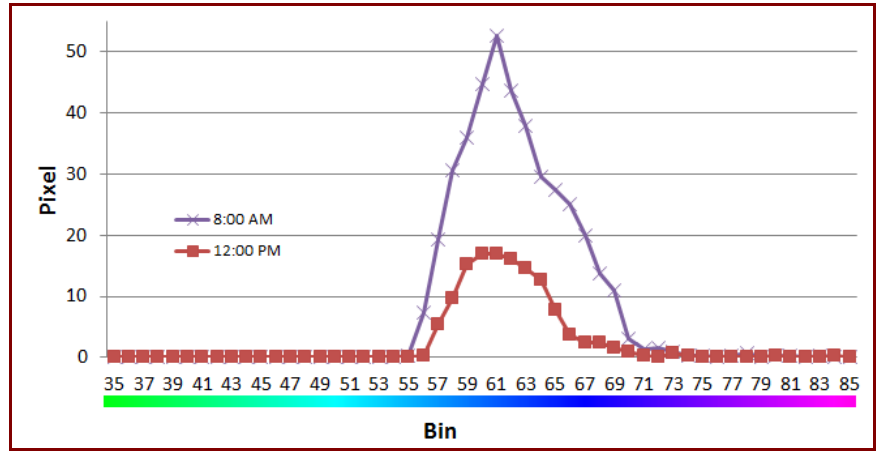

Fig 2 Hue histogram for the segmented images

The higher amount of the blue colored hue properties generated at $8.00 \mathrm{am}$ is due to the minimal sunlight emission, hence producing stronger blue ambient lighting effect for FFB images captured in the morning. The strong effect of blue ambient lighting, which occurs in the morning and during overcast will reduce the perception of the actual oil palm FFB surface color particularly for FFB ripeness observation in the conventional method of oil palm harvesting. As the time passes, the blue ambient lighting effect will be slowly diminishing since the sun gradually travels perpendicular to the horizon. At 12:00 pm, the sun is now perpendicular to the horizon and radiates at maximum intensity thus reducing the blue ambient lighting effect for FFB images captured at noon.

In order to reduce the effect of blue ambient lighting generated on the FFB images by the irregular outdoor lighting condition, the oil palm FFB is radiated with the artificial lighting before the FFB image is captured. The reduction of the blue ambient lighting effect in outdoor image analysis application is crucial, as the occurrences of the blue ambient lighting effect during FFB image acquisition will change the pixel values of the captured images color properties.

The optimum distance between the artificial light source and the oil palm FFB is determined by gradually shifting the artificial light source from the initial distance of 5 inches up to 12 inches of distance from the FFB. The results are shown in Fig. 3. From the observation, the application of artificial lighting to the oil palm FFB before capturing the FFB image has significantly reduce the effect of blue ambient lighting generated from the skylight. The minimum pixel values of the blue colored hue properties is recorded at 10 inches of distance with 19 pixels at peak, close to the pixels generated by FFB images captured at 12:00pm when the blue ambient lighting effect is at minimal. As the artificial light source is gradually moved away from the FFB, the pixels of the blue colored hue properties is gradually increasing with maximum pixel values of 20 pixels and 25 pixels recorded at 11 inches and 12 inches of distance.

Among all of the applied distances, the blue colored hue properties for FFB images captured at 11 inches of distance shows the most symmetrical in pixel distribution comparable to pixel distribution generated by the FFB images captured at 12:00 pm as shown in Fig. 3 (g). While the artificial lighting generate the highest improvement against the blue ambient lighting effect at 10 inches of distance, the overall pixel distribution is asymmetrical with a significant decrease in pixels at bin 61 to bin 67 as shown in Fig. 3 (f) which change the overall pixel distribution. Therefore, the distance of 11 inches between the FFB and the artificial light source is selected for the proposed artificial lighting system in order to produce an optimal correction against the blue ambient lighting effect.

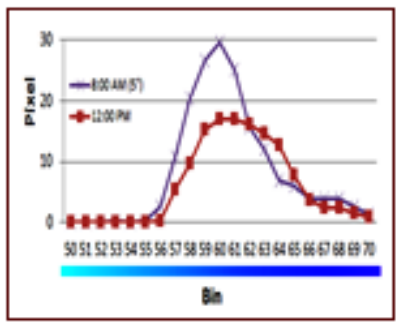

(a) 5 inches.

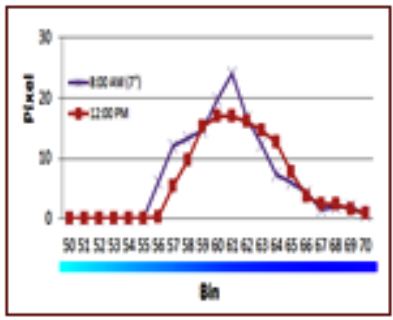

(c) 7 inches.

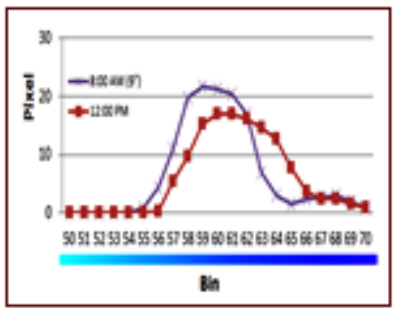

(e) 9 inches.

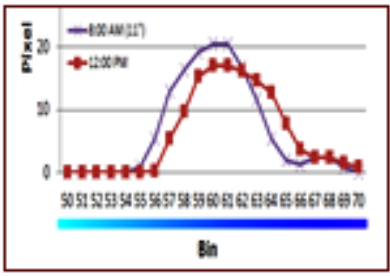

(g) 11 inches.

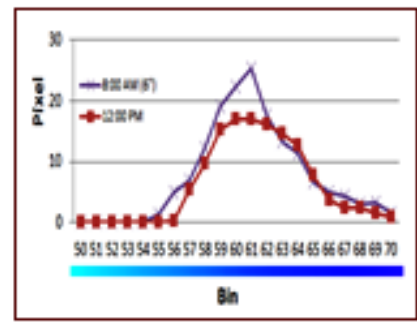

(b) 6 inches.

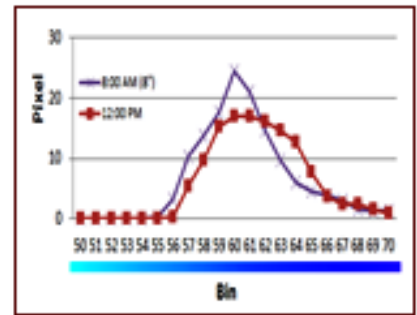

(d) 8 inches.

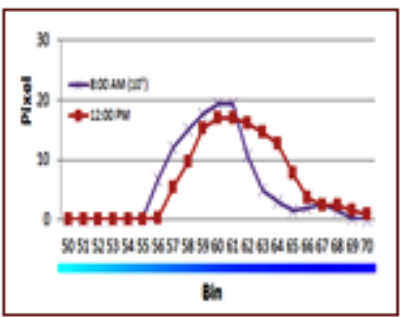

(f) 10 inches.

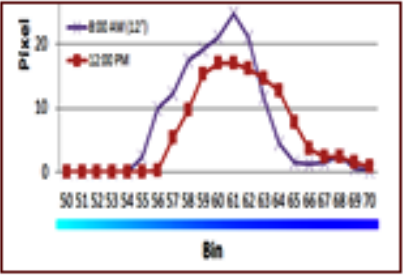

(h) 12 inches.
Fig. 3 The graph of hue histogram for FFB images at different artificial light source distance. 
In order to investigate the significance of artificial lighting effect on each FFB ripeness categories, the population mean for each FFB ripeness categories for all three artificial lighting modes are compared to each other by performing an independent two-sample-t-test analysis using Minitab software at $95 \%$ of confidence level. The acquired p-values for each combination of different FFB ripeness categories at three different lighting mode factors of Off, Auto, and On are displayed in Table 1 until Table 3.

TABLE I

p-VAlues Using OfF MOde

\begin{tabular}{lcccc}
\hline \hline Category & Unripe & Underripe & Ripe & Overripe \\
Unripe & - & 0.000 & 0.001 & 0.000 \\
Underripe & - & - & 0.650 & 0.308 \\
Ripe & - & - & - & 0.178 \\
Overripe & - & - & - & - \\
\hline \hline
\end{tabular}

TABLE II

p-VALUES USING AUTOMODE

\begin{tabular}{lcccc}
\hline \hline Category & Unripe & Underripe & Ripe & Overripe \\
\hline Unripe & - & 0.011 & 0.000 & 0.000 \\
Underripe & - & - & 0.065 & 0.000 \\
Ripe & - & - & - & 0.014 \\
Overripe & - & - & - & - \\
\hline \hline
\end{tabular}

TABLE III

p-VALUeS Using ON MOdE

\begin{tabular}{lcccc}
\hline \hline Category & Unripe & Underripe & Ripe & Overripe \\
Unripe & - & 0.000 & 0.000 & 0.000 \\
Underripe & - & - & 0.090 & 0.001 \\
Ripe & - & - & - & 0.045 \\
Overripe & - & - & - & - \\
\hline \hline
\end{tabular}

By referring to Table 1 until Table 3, the hue mean populations for the Off lighting mode shows a significant difference for FFB ripeness combinations of UnripeUnderripe, Unripe-Ripe, and Unripe-Overripe with the pvalues smaller than 0.05 , while for FFB ripeness combinations of Underripe-Ripe, Underripe-Overripe, and Ripe-Overripe shows a non-significant difference in hue mean population. The results show that the distinction between four FFB ripeness categories of Unripe, Underripe, Ripe, and Overripe is only significant for comparison between the Unripe against other ripeness categories, as the Unripe FFB has a much higher blue colored hue properties making it more distinctive than other ripeness categories. While FFB ripeness categories of Underripe, Ripe, and Overripe, the difference is not significant, as the effect of blue ambient lighting from the skylight had reduced the distinction between all three ripeness categories observed in the outdoor lighting environment.

Meanwhile, for Auto and On lighting modes the effect of artificial lighting had significantly improved the distinction between FFB ripeness categories of Underripe-Overripe and Ripe-Overripe with the p-values for each of combinations less than 0.05 compared to Off lighting mode. However, the distinction between the Underripe-Ripe FFB ripeness categories is not significant with the p-values for both lighting factors at 0.065 (Auto mode) and 0.090 (On mode). Although the distinction between the Underripe-Ripe FFB ripeness categories is not significant, the use of artificial lighting had improved the distinction between these two categories compared to FFB images captured without the artificial lighting (Off mode). The use of Auto lighting mode also provides a better distinction between the Underripe and Ripe FFB ripeness categories with the p-value of 0.065 compared to On lighting mode at 0.090 since the Auto lighting mode varies the artificial lighting intensity according to outdoor lighting environment thus producing a less glaring effect onto the FFB surface.

These results suggest that the use of artificial lighting in outdoor image analysis applications will significantly improve the distinction between the Unripe-Underripe, Unripe-Ripe, Underripe-Overripe, and Ripe-Overripe FFB ripeness categories, while providing a better discrimination between underripe and Ripe FFB ripeness categories compared to FFB images captured without the artificial lighting.

Even though the application of an artificial lighting in an outdoor image analysis application for both Auto and On lighting modes provide a better discrimination between FFB maturity levels, continuous radiation from the artificial lighting at maximum light intensity will generate high power consumption. The On lighting treatment draws $0.71 \mathrm{~A}$ of electrical current according to the current sensor measurement for each of lighting session. At $6.6 \mathrm{~V}$ of LED operating voltage, the On lighting mode constantly consume around 4.70 Watt of electrical power on each lighting session. While for the Auto lighting mode, the amount of electrical current draws on each of the lighting session varies according to the measured outdoor light intensity. The overall power consumption for the Auto lighting mode is displayed in Table 4.

By referring to Table 4, it can be observed that the power consumption of the Auto lighting mode varies from 3.64 Watt to 4.55 Watt at each of lighting session, which results in around $3.19 \%$ to $22.55 \%$ of improvement in power consumption at each lighting session compared to the On lighting mode. Therefore, the application of LDR light sensor for the Auto lighting treatment has provide a better power consumption on the proposed artificial lighting system, while still deliver a better discrimination between FFB maturity levels in the outdoor image analysis application.

\section{CONCLUSION}

The application of artificial lighting in outdoor image analysis of oil palm FFB will improve the distinction of FFB color properties by significantly reduce the effect of blue ambient lighting generated by the skylight on every FFB ripeness categories. The system uses LDR as a light sensor in controlling the artificial light intensity automatically, which 
improve the power consumption by $22.5 \%$. The optimum distance between the oil palm FFB and the artificial light source is 11 inches. The use of artificial lighting in outdoor image analysis applications will significantly improve the distinction between the Unripe-Underripe, Unripe-Ripe, Underripe-Overripe, and Ripe-Overripe FFB ripeness categories, while providing a better discrimination between underripe and Ripe FFB ripeness categories compared to FFB images captured without the artificial lighting.

$$
\text { TABLE IV }
$$

POWER Consumption OF THe Auto Lighting Mode

\begin{tabular}{|c|c|c|c|c|c|c|c|c|c|c|}
\hline \multirow{2}{*}{$\begin{array}{c}\text { FFB } \\
\text { Maturity }\end{array}$} & \multirow[b]{2}{*}{ Sample } & \multicolumn{9}{|c|}{ Power Consumption (Watt) } \\
\hline & & 08:00 & 09:00 & 10:00 & 11:00 & 12:00 & 01:00 & 02:00 & 03:00 & 04:00 \\
\hline \multirow[t]{3}{*}{ Level } & & AM & AM & AM & AM & PM & PM & PM & PM & PM \\
\hline & 1 & 4.33 & 4.11 & 4.11 & 4.11 & 4.11 & 4.11 & 4.11 & 4.06 & 3.97 \\
\hline & 2 & 4.32 & 4.06 & 4.11 & 4.11 & 3.97 & 4.33 & 4.18 & 4.33 & 3.97 \\
\hline \multirow[t]{5}{*}{ Unripe } & 3 & 4.55 & 4.33 & 4.20 & 4.33 & 4.11 & 4.17 & 4.11 & 4.55 & 4.14 \\
\hline & 4 & 4.64 & 4.32 & 4.11 & 4.55 & 4.33 & 3.64 & 4.46 & 4.33 & 4.03 \\
\hline & 5 & 4.33 & 4.23 & 4.11 & 4.18 & 4.33 & 4.11 & 4.09 & 4.32 & 4.33 \\
\hline & 1 & 4.64 & 4.55 & 4.33 & 4.11 & 4.11 & 3.64 & 4.33 & 4.32 & 4.33 \\
\hline & 2 & 4.55 & 4.33 & 4.55 & 4.20 & 4.11 & 4.33 & 4.55 & 4.33 & 4.33 \\
\hline \multirow[t]{5}{*}{ Underripe } & 3 & 4.55 & 4.33 & 4.33 & 4.06 & 4.11 & 4.11 & 4.17 & 4.18 & 4.14 \\
\hline & 4 & 4.33 & 4.33 & 4.11 & 4.18 & 4.11 & 3.97 & 4.55 & 4.46 & 4.03 \\
\hline & 5 & 4.32 & 4.32 & 4.23 & 4.33 & 4.20 & 4.33 & 4.09 & 4.11 & 3.97 \\
\hline & 1 & 4.55 & 4.17 & 4.55 & 4.11 & 4.11 & 4.33 & 4.09 & 4.33 & 4.14 \\
\hline & 2 & 4.33 & 4.23 & 4.11 & 3.97 & 4.33 & 4.32 & 4.11 & 4.18 & 4.03 \\
\hline \multirow[t]{5}{*}{ Ripe } & 3 & 4.32 & 4.09 & 4.18 & 4.32 & 3.64 & 4.23 & 4.32 & 4.18 & 4.14 \\
\hline & 4 & 4.64 & 4.32 & 4.33 & 4.18 & 3.64 & 4.11 & 4.33 & 4.46 & 4.33 \\
\hline & 5 & 4.32 & 4.33 & 4.11 & 4.06 & 4.33 & 4.55 & 4.11 & 4.20 & 3.97 \\
\hline & 1 & 4.64 & 4.33 & 4.46 & 4.33 & 4.33 & 4.17 & 3.97 & 4.33 & 4.03 \\
\hline & 2 & 4.64 & 4.11 & 4.11 & 4.23 & 3.64 & 4.33 & 4.33 & 4.18 & 4.33 \\
\hline \multirow[t]{3}{*}{ Overripe } & 3 & 4.33 & 4.33 & 4.32 & 4.33 & 4.11 & 3.97 & 4.09 & 4.55 & 4.03 \\
\hline & 4 & 4.32 & 4.32 & 4.33 & 4.20 & 4.06 & 4.11 & 4.11 & 4.18 & 3.97 \\
\hline & 5 & 4.55 & 4.55 & 4.11 & 4.17 & 4.11 & 4.33 & 4.33 & 4.46 & 4.14 \\
\hline
\end{tabular}

\section{ACKNOWLEDGMENT}

This research was supported by Research Universiti Grant, in title of "Development of Intelligent Vision System on Portable Platform for The Assessment of Oil Palm Fresh Fruit Bunches (FFB)", account number 1001/PELECT/814266 from Universiti Sains Malaysia.

\section{REFERENCES}

[1] Corley, R. H. V. \& Tinker, P. B. H., 2003. The Oil Palm. Oxford, UK: Blackwell Science Ltd. https://doi.org/10.1002/9780470750971

[2] Rahman, A. K. A., Abdullah, R., Shariff, F. M. \& Simeh, M. A., 2008. The Malaysian Palm Oil Supply Chain: The Role of the Independent
Smallholders. Oil Palm Industry Economic Journal (Vol. 8(2)/2008), pp. 17-27.

[3] Abdullah, M. Z., Guan, L. C. \& Azemi, B. M. N. M., 2001. Stepwise Discriminant Analysis for Colour Grading of Oil Palm using Machine Vision System. Institution of Chemical Engineers Trans IChemE, 79(Part C), pp. 223-231 https://doi.org/10.1205/096030801753252298

[4] Jaffar, A. Photogrammetric Grading of Oil Palm Fresh Fruit Bunches. International Journal of Mechanical \& Mechatronics Engineering (IJMME), IX(10), pp. 18-24.2009

[5] May, Z. \& Amaran, M. H. Automated Oil Palm Fruit Grading System using Artificial Intelligence. International Journal of Video \& Image Processing and Network Security IJVIPNS-IJENS, XI(03), pp. 30-35,2011.

[6] Ismail, W. I. W. \& Razali, M. H., Outdoor Colour Recognition System for Oil Palm Fresh Fruit Bunches (FFB). International Journal of Machine Intelligence, pp. 01-10, 2010b.

[7] Chen, H. \& Mori, H.. Sign Pattern Detection on Shaded Road. Tsukuba, Japan, Intelligent Robots and Systems '89. The Autonomous Mobile Robots and Its Applications. IROS '89. Proceedings., IEEE/RSJ International Workshop, 1989.

[8] Mori, H., Kobayashi, K., Ohtuki, N. \& Kotani, S.,I . Color Impression Factor: An Image Understanding Method for Outdoor Mobile Robots. Grenoble, Intelligent Robots and Systems, 1997.

[9] Ismail, W. I. W., Bardaie, M. Z. \& Hamid, A. M. A., 2000. Optical Properties for Mechanical Harvesting of Oil Palm FFB. Journal of Oil Palm Research, 12(2), pp. 38-45, 2000.

[10] Junkwon, P. Potental Appllication of Color and Hyperspectral Images for Estimation of Weight and Ripeness of Oil Palm (Elaieis Guineensis Jacq. var. tenera). Agricultural Information Research, 18(2), pp. 72-81, 2009. https://doi.org/10.3173/air.18.72

[11] Jamil, N., Mohamed, A. \& Abdullah, S.. Automated Grading of Palm Oil Fresh Fruit Bunches (FFB) Using Neuro-Fuzzy Technique. Malacca, Malaysia, International Conference of Soft Computing and Pattern Recognition, 2009.

[12] Slaughter, D. C. \& Harrell, R. C.. Color Vision in Robotic Fruit Harvesting. Transaction of the ASABE, 30(4), pp. 1144-1148, 1987. https://doi.org/10.13031/2013.30534

[13] Blandini, G. \& Levi, P.. First Approaches to Robot Utilisation for Automatic Citrus harvesting. Agricultural Engineering, Volume III, pp. 1903-1907,1989.

[14] Singh, S. Comprehensive Automation for Specialty Crops: Year 1 results and lessons learned. Intel Serv Robotics, Issue Special Issue, pp. 245262,2010

[15] Wang, Q., Wang, H., Xie, L. \& Zhang, Q. Outdoor Color Rating of Sweet Cherries Using Computer Vision. Computers and Electronics in Agriculture, Issue 87, pp. 113-120, 2012. https://doi.org/10.1016/j.compag.2012.05.010

[16] Koselka, T. Robot scout for tree fruit. Washington Tree Fruit Research Commission,2009.

[17] Bridgelux, Inc. Bridgelux LS Array Series Product Data Sheet DS14, Livermore, CA: Bridgelux, Inc., 2011.

[18] Fadilah, N. et al. Intelligent Color Vision System for Ripeness Classification of oil Palm Fresh Fruit Bunch. Sensors, XII(10), pp. 14179$14195,2012$. https://doi.org/10.3390/s121014179

[19] Fadilah, N., Saleh, J. M., Ibrahim, H. \& Halim, Z. A., . Oil Palm Fresh Fruit Bunch Ripeness Classification using Artificial Neural Network. Kuala Lumpur, International Conference on Intelligent and Advanced Systems, 2012. https://doi.org/10.1109/icias.2012.6306151

[20] Fadilah, N. \& Saleh, J. M.. Color Feature Extraction of Oil Palm Fresh Fruit Bunch Image for Ripeness Classification. Kuala Lumpur, World Scientific and Engineering Academy Society (WSEAS) Press, 2014. 